\title{
Cause Cannot be Traced to Device
}

National Cancer Institute

\section{Source}

National Cancer Institute. Cause Cannot be Traced to Device. NCI Thesaurus. Code C139470.

The adverse event that occurred is not attributable to a device. 